\title{
NonLinear Control via Input-Output Feedback Linearization of a Robot Manipulator
}

\author{
Wafa Ghozlane*, Jilani Knani \\ Laboratory of Automatic Research LA.R.A, Department of Electrical Engineering, National School of Engineers of Tunis ENIT, \\ University of Tunis ElManar, 1002 Tunis, Tunisia.
}

\begin{tabular}{l} 
A R T I C L E I N F O \\
\hline Article history: \\
Received: 08 September, 2018 \\
Accepted: 13 October, 2018 \\
Online: 18 October, 2018 \\
\hline
\end{tabular}

Keywords:

Input-Output Feedback

linearization

Nonlinear system

Robot manipulator

MIMO nonlinear system

SISO linear systems

$P D$ controller

\begin{abstract}
A B S T R A C T
This paper presents the input-output feedback linearization and decoupling algorithm for control of nonlinear Multi-input Multi-output MIMO systems. The studied analysis was motivated through its application to a robot manipulator with six degrees of freedom. The nonlinear MIMO system was transformed into six independent single-input single-output SISO linear local systems. We added PD linear controller to each subsystem for purposes of stabilization and tracking reference trajectories, the obtained results in different simulations shown that this technique has been successfully implemented.
\end{abstract}

\section{Introduction}

In recent years, Feedback linearization has been attracted a great deal of interesting research. It's an approach designed to the nonlinear control systems, which based on the idea of transforming nonlinear dynamics into a linear form. The base idea of this technique is to algebraically transform a nonlinear dynamics system into a totally or partially linear one, so that linear control techniques can be applied. This notion can be used for both stabilization and tracking control objectives of SISO or MIMO systems, and has been successfully applied to a number of practical nonlinear control problems such as [1-4].

In fact, this technique has been successfully implemented in several faisable applications of control, such as industrial robots, high performance aircraft, helicopters and biomedical dispositifs, more tasks used the methodology are being now well advanced in industry [5-6].

In this case, we applied this technique to lead the control for each joint of a robot manipulator that is has six degrees of freedom, which the equations of motion form a nonlinear, complex dynamic and multivariable system, then, we elaborated a PD linear controller for each decoupled linear subsystem to control

*Corresponding Author: Wafa Ghozlane, Email: ghozlanewafa@yahoo.fr the angular position of each joint of this robot arm for stabilization and tracking purposes. The obtained results in different simulations shown the efficiency of the derived approach [7].

This paper is organized as follows: It is divided into five sections. In Section 2, a description of the input-output feedback linearization approach is detailed. In Section 3, a simplified dynamic model of a robot manipulator with six degrees of freedom is presented, the input-output feedback linearization method is applicated to the above robot and the construction of linear PD controller is derived. In Section 4, the simulation results are presented. Finally, the conclusion was elaborated in Section 5.

\section{Input-Output feedback linearization for MIMO nonlinear system.}

In this section, we discussed the approach of input-output feedback linearization of nonlinear systems, the central goal of feedback linearization is to design a nonlinear control law as assumed that the inner loop control is, in the most suitable case, precisely linearizes the nonlinear system after appropriate state space modification of coordinates [1]. The developer can then build an outer loop control in the new coordinates to obtain a linear relation between the output $\mathrm{Y}$ and the input $\mathrm{V}$ and to satisfy the traditional control design specifications such as tracking, disturbance rejection, as shown in Figure 1. 


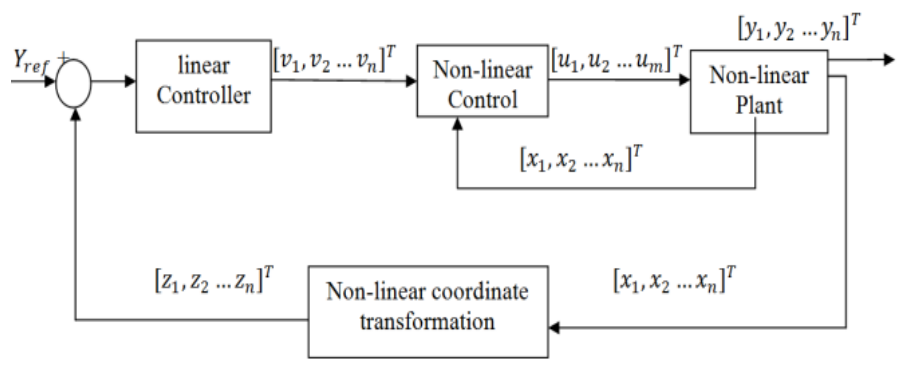

Figure 1. Structure of Input-Output Feedback Linearization Approach.

The basic condition for using feedback linearization method is nonlinear dynamic MIMO of $n$-order with $p$ number of inputs and outputs described in the affine form;

$$
\left\{\begin{array}{c}
\dot{X}(t)=f(X(t))+\sum_{i=1}^{p} g_{i}(X(t)) U_{i}(t) \\
Y_{i}(t)=h_{i}(X(t)) \\
i=1,2, \ldots p
\end{array}\right.
$$

Where,

$X=\left[x_{1}, x_{2} \ldots x_{n}\right]^{T} \in R^{n}:$ is the state vector.

$U=\left[u_{1}, u_{2} \ldots u_{p}\right]^{T} \in R^{p}:$ is the control input vector.

$Y=\left[y_{1}, y_{2} \ldots y_{p}\right]^{T} \in R^{p}:$ is the output vector, $f(X)$, and $g_{i}(X)$ :

are n-dimentional smooth vector fields.

$h_{i}(X)$ : is smooth nonlinear functions, with $\mathrm{i}=1,2 \ldots \mathrm{n}$.

\section{Theorem1:}

Let f: $\mathcal{R}^{\mathrm{n}} \rightarrow \mathcal{R}^{\mathrm{n}}$ represent a smooth vector field on $\mathcal{R}^{\mathrm{n}}$ and let

$\mathrm{h}: \mathcal{R}^{\mathrm{n}} \rightarrow \mathcal{R}^{\mathrm{n}}$ represent a scalar function. The Lie Derivative of $\mathrm{h}$, with respect to $f$, denoted $\mathrm{L}_{\mathrm{f}} \mathrm{h}$, is defined as [1-2].

$$
L_{f} h=\frac{\partial h}{\partial x} f(x)=\sum_{i=1}^{p} \frac{\partial h}{\partial x_{i}} f_{i}(x)
$$

The Lie derivative is the directional derivative of $h$ in the direction of $\mathrm{f}(\mathrm{x})$, in an equivalent way, the inner product of the gradient of $h$ and $f$. We defined by $L_{f}{ }^{2} h$ the Lie Derivative of $L_{f} h$ with respect to $\mathrm{f}$ :

$$
L_{f}^{2} h=L_{f}\left(L_{f} h\right)
$$

In general we define:

$$
L_{f}{ }^{k} h=L_{f}\left(L_{f}{ }^{k-1} h\right) \text { for } k=1, \ldots, p
$$

with $L_{f}^{0} h=h$

Theorem2:

The function $\Phi: \mathcal{R}^{\mathrm{n}} \rightarrow \mathcal{R}^{\mathrm{n}}$ defined in a region $\Omega \subset \mathcal{R}^{\mathrm{n}}$ he is called difeomorphisme if it checks the following conditions:
Firstly, a diffeomorphism is a differentiable function whose inverse exists and is also differentiable. Second, we should assume that both the function and its inverse to be infinitely differentiable, such functions are usually referred to as $\mathbb{C} \infty$ diffeomorphisms [3-4].

The diffeomorphism is used to transform one nonlinear system in another nonlinear system by making a change of variables of the form:

$$
z=\Phi(x)
$$

Where $\Phi(\mathrm{x})$ represents $\mathrm{n}$ variables;

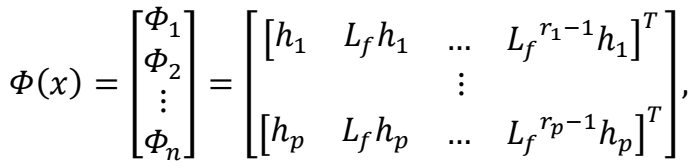

$$
\begin{aligned}
& x=\left[x_{1}, x_{2} \ldots x_{n}\right]^{T}
\end{aligned}
$$

The goal is to obtain a linear relation between the inputs and the outputs by differentiating the outputs $y_{j}$ until the inputs appear. Suppose that $r_{j}$ is the smallest integer such that fully one of the inputs appears in $y_{j}^{\left(r_{j}\right)}$ using this expression:

$$
\begin{gathered}
y_{j}\left(r_{j}\right)=L_{f}{ }^{r_{j}} h_{j}(x)+\sum_{i=1}^{p} L_{g_{i}}\left(L_{f}\left(r_{j-1}\right) h_{j}(x)\right) u_{i} \\
i, j=1,2, \ldots p
\end{gathered}
$$

Where, $L_{f}{ }^{i} h_{j}$ and $L_{g}{ }^{i} h_{j}$ : Are the $i^{t h}$ Lie derivatives of $h_{\mathrm{j}}(\mathrm{x})$ respectively in the direction of $f$ and $g$.

$$
L_{f} h_{j}(x)=\frac{\partial h_{j}}{\partial x} f(x), L_{g} h_{j}(x)=\frac{\partial h_{j}}{\partial x} g_{i}(x)
$$

$r_{j}$ : is the relative degree corresponding to the $y_{j}$ output, it's the number of necessary derivatives so that at least one of the inputs appear in the expression [5].

If expression $L_{g} h_{j}(x)=0$, for all $\mathrm{i}$, then the inputs have not appeared in the derivation and it's necessary to continu the derivation of the output $y_{j}$.

The system (1) has the relative degree (r) if it satisfies:

$$
\left\{\begin{array}{c}
L_{g_{i}} L_{f}{ }^{k} h_{j}=0 \quad 0<k<r_{j-1}, 0 \leq i \leq n, 0 \leq j \leq n \\
L_{g_{i}} L_{f}{ }^{k} h_{j} \neq 0 \quad k=r_{j-1}
\end{array}\right.
$$

The total relative degree (r) was considered as the sum of all the relative degrees obtained using (7) and must be less than or equal to the order of the system (10):

$$
r=\sum_{j=1}^{n} r_{j} \leq n
$$

To find the expression of the nonlinear control law $U$ that allows to make the relationship linear between the input and the output [6], the expression (2) is rewritten in matrix form as: 


$$
\begin{aligned}
& {\left[y_{1}^{r_{1}} \ldots y_{p}{ }^{r_{p}}\right]^{T}=\propto(x)+\beta(x) . U} \\
& V=\left[\begin{array}{llll}
v_{1} & v_{2} & \ldots & v_{p}
\end{array}\right]^{T}=\left[y_{1}^{r_{1}} \ldots y_{p}^{r_{p}}\right]^{T}
\end{aligned}
$$

Where:

$$
\begin{aligned}
& \propto(x)=\left[\begin{array}{c}
L_{f}{ }^{r_{1}} h_{1}(x) \\
\cdot \\
\cdot \\
L_{f}{ }^{r_{p}} h_{p}(x)
\end{array}\right] \\
\beta & =\left[\begin{array}{cccc}
L_{g_{1}}\left(L_{f}{ }_{f}^{\left(r_{1}-1\right)} h_{1}(x)\right) & L_{g_{2}}\left(L_{f}{ }^{\left(r_{1}-1\right)} h_{1}(x)\right) & \ldots & L_{g_{p}}\left(L_{f}{ }^{\left(r_{1}-1\right)} h_{1}(x)\right) \\
L_{g_{1}}\left(L_{f}{ }^{\left(r_{2}-1\right)} h_{2}(x)\right) & L_{g_{2}}\left(L_{f}{ }^{\left(r_{2}-1\right)} h_{2}(x)\right) & \ldots & L_{g_{p}}\left(L_{f}{ }^{\left(r_{2}-1\right)} h_{2}(x)\right) \\
\cdot & \cdot & \ldots & \vdots \\
\cdot & \cdot & & \vdots \\
L_{g_{1}}\left(L_{f}{ }^{\left(r_{p}-1\right)} h_{p}(x)\right) & L_{g_{2}}\left(L_{f}{ }^{\left(r_{p}-1\right)} h_{p}(x)\right) & \ldots & L_{g_{p}}\left(L_{f}{ }^{\left(r_{p}-1\right)} h_{p}(x)\right)
\end{array}\right](14)
\end{aligned}
$$

If $\beta(\mathrm{x})$ is not singular, then it is possible to define the input transformation "the nonlinear control law " which has this form:

$$
\begin{gathered}
U=\beta(x)^{-1} \cdot(-\propto(x)+V) \\
V=\left[\begin{array}{llll}
v_{1} & v_{2} & \ldots & v_{p}
\end{array}\right]^{T} \\
U=\left[\begin{array}{llll}
u_{1} u_{2} & \ldots & u_{p}
\end{array}\right]^{T} \\
y_{j}^{r_{j}}=v_{j}
\end{gathered}
$$

where

$\mathrm{V}$ : Is the new input vector. is called a decoupling control law

$\beta(x)$ :Is the invertible ( $\mathrm{pxp}$ ) matrix. Is called a decoupling matrix of the system.

\subsection{Non-linear coordinate transformation:}

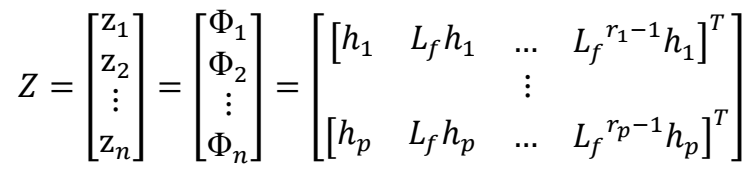

By applying the linearizing law to the system,we can transforme the nonlinear system into linear form [7-8]:

$$
\left\{\begin{array}{c}
\dot{\mathrm{Z}}=\mathrm{Az}+\mathrm{BV} \\
\mathrm{Y}=\mathrm{CZ}
\end{array}\right.
$$

With,

$$
\begin{gathered}
\mathrm{A}=\left[\begin{array}{ccc}
\mathrm{A}_{\mathrm{r}_{1}} & \ldots & 0 \\
\ldots & \ldots & \ldots \\
0 & \ldots & \mathrm{A}_{\mathrm{r}_{\mathrm{p}}}
\end{array}\right], \mathrm{B}=\left[\begin{array}{ccc}
\mathrm{B}_{\mathrm{r}_{1}} & \ldots & 0 \\
\ldots & \ldots & \ldots \\
0 & \ldots & \mathrm{B}_{\mathrm{r}_{\mathrm{p}}}
\end{array}\right], \\
\mathrm{C}=\left[\begin{array}{ccc}
\mathrm{C}_{\mathrm{r}_{1}} & \ldots & 0 \\
\ldots & \ldots & \ldots \\
0 & \ldots & \mathrm{C}_{\mathrm{r}_{\mathrm{p}}}
\end{array}\right]
\end{gathered}
$$

And,

$$
\mathrm{A}_{\mathrm{r}_{\mathrm{i}}}=\left[\begin{array}{cccc}
0 & 1 & \ldots & 0 \\
0 & 0 & \ldots & 0 \\
\cdots & \ldots & \ldots & \ldots \\
0 & 0 & \ldots & 1 \\
0 & 0 & \ldots & 0
\end{array}\right] \in \mathcal{R}^{\mathrm{r}_{\mathrm{i}} \times \mathrm{r}_{\mathrm{i}}} ; \mathrm{B}_{\mathrm{r}_{\mathrm{i}}}=\left[\begin{array}{c}
0 \\
\vdots \\
1
\end{array}\right] \in \mathcal{R}^{\mathrm{r}_{\mathrm{i}}}
$$

$$
\mathrm{C}_{\mathrm{r}_{\mathrm{i}}}=\left[\begin{array}{llll}
1 & 0 & \ldots & 0
\end{array}\right] \in \mathcal{R}^{\mathrm{r}_{\mathrm{i}}}
$$

\subsection{Design of the new control vector $V$ :}

The vector $\mathrm{v}$ is designed to according the control objectives, for the tracking problem considered, it must satisfy:

$$
\begin{gathered}
v_{j}=y_{d_{j}}{ }^{r_{j}}+K_{r_{j-1}}\left(y_{d_{j}}{ }^{r_{j-1}}-y_{j}{ }^{r_{j-1}}\right)+\cdots \\
+K_{1}\left(y_{d_{j}}-y_{j}\right) ; \\
1 \leq j \leq p
\end{gathered}
$$

where,

$\left\{y_{d_{j}}, y_{d_{j}}{ }^{2}, \ldots, y_{d_{j}}{ }^{r_{j-1}, y_{d_{j}}}{ }^{r_{j}}\right\}$ denote the imposed reference trajectories for the different outputs. If the $K_{i}$ are chosen so that the polynomial [9-10];

$s^{r_{j}}+K_{r_{j-1}} s^{r_{j-1}}+\cdots+K_{2} s+K_{1}=0$ are Hurwitz (has roots with negative real parts). Then it can be shown that the error $e_{j}(t)=y_{d_{j}}(t)-y_{j}(t)$, satisfied $\lim _{t \rightarrow \infty} e_{j}(t)=0$.

\section{Input-Output feedback linearization approach applied to a robot manipulator with six degrees of freedom}

\subsection{Dynamic modeling of a robot manipulator}

In this section, we have applied the proposed approach to a dynamic multivariable system which represent a robot arm with six degrees of freedom "EPSON C4". This is an open chain kinematic manipulator robot consisting of seven rigid bodies interconnected by six revolute joints $n=6$ as [11]. So, deriving the motion of robot is a complex task due to the nonlinearities present in this system and the large number of degrees of freedom [12-13]. Then, it is essential to understand exactly the dynamics of this interconnected chain of rigid bodies, to detemine the inverse dynamics model such as relation (19), we analyzed the evolution of motion of this mechanical non linear system by using the Euler-Lagrange equations, which is represented by the equation (20).

$$
\begin{gathered}
\Gamma=\mathrm{f}\left(\mathrm{q}, \dot{\mathrm{q}}, \ddot{\mathrm{q}}, \mathrm{f}_{\mathrm{e}}\right) \\
\Gamma_{\mathrm{i}}=\sum_{\mathrm{j}=1}^{\mathrm{n}} \mathrm{d} \frac{\left(\frac{\partial \mathrm{L}_{\mathrm{j}}}{\partial \mathrm{q}_{1}}\right)}{\mathrm{dt}}-\frac{\partial \mathrm{L}_{\mathrm{j}}}{\partial \mathrm{q}_{\mathrm{i}}} \mathrm{i}, \mathrm{j}=1, \ldots, \mathrm{n}
\end{gathered}
$$

where $\Gamma, q, \dot{q}, \ddot{q}$ and $f_{e}$ depicting Torques, articular positions, velocities, accelerations and the external force.

$L_{j}$ : Defines the lagrangian of the $j^{\text {th }}$ link, which is the difference of the kinetic and potential energy, equal to $E_{j}-U_{j}$.

$E_{j}$ and $U_{j}$ : Define the kinetic and the potential energies of the $j^{\text {th }}$ link. 


\section{The calculation of the kinetic energy;}

In this section, we have calculated the total kinetic energy of the system which depends on the configuration and joint velocities, such that [13], then, it was described by the equation (21).

$$
E=\sum_{j=1}^{n} E_{j}
$$

$E_{j}$ : means the kinetic energy of the link $C_{j}$, which can be formulated such that (24). Firstly, we have calcuated the linear velocity and the angular velocity using the equations (22) and (23).

$$
\begin{gathered}
{ }_{j}^{j} V={ }_{j-1}^{j} A\left({ }_{j-1}^{j-1} V+{ }_{j-1}^{j-1} W \times P_{j}^{j-1}\right)+\sigma_{j} \dot{q}_{j} a_{j} \\
{ }_{j}^{j} W={ }_{j-1}^{j} A \times{ }_{j-1}^{j-1} W+\bar{\sigma}_{j} \times \dot{q}_{j} \times a_{j}
\end{gathered}
$$

${ }_{j-1}^{j-1} V$ : The linear velocity, it is the derivative of the position vector $P_{j}^{j-1}$.

${ }_{j-1}^{j-1} W:$ The angular velocity.

The initial conditions for a robot which the base is fixed, are

${ }_{0}^{0} \mathrm{~V}=0$ and ${ }_{0}^{0} W=0$.

Then,we have expressed these relations (22) and (23) in Equation (24) as.

$$
E_{j}=\frac{1}{2}\left[{ }_{j}^{j} W^{T}{ }_{j}^{j} J_{j}^{j} W+M_{j}{ }_{j}^{j} V^{T}{ }_{j}^{j} V+2 M S_{j}^{T}\left({ }_{j}^{j} V \wedge{ }_{j}^{j} W\right)\right]
$$

where

$a_{j}$ :is the unit vector along axis $z_{j}$.

$\mathrm{M}_{\mathrm{j}},{ }_{\mathrm{j}}^{\mathrm{j}} \mathrm{MS}$ et ${ }_{\mathrm{j}}^{\mathrm{j}} \mathrm{J}$ :are the inertial standard parameters.

$\mathrm{M}_{\mathrm{j}}$ : is the mass of link $C_{j}$.

${ }_{\mathrm{j}}^{\mathrm{j}} \mathrm{MS}$ :design the first moments of inertia of link $C_{j}$ about the origin of the frame $R_{j}$ It is equal to ${ }_{\mathrm{j}}^{\mathrm{j}} \mathrm{MS}=\left[\mathrm{MX}_{\mathrm{j}} \mathrm{MY}_{\mathrm{j}} \mathrm{MZ}_{\mathrm{j}}\right]^{\mathrm{T}}$.

${ }_{\mathrm{j}}^{\mathrm{j}} \mathrm{J}$ : is the inertial tensor matrix $(3 \times 3)$ of link $\mathrm{C}_{\mathrm{j}}$ with respect to the frame $R_{j}$, it is expressed by the matrix (25).

$$
{ }_{j}^{j} J==\left[\begin{array}{ccc}
I_{X X_{j}} & I_{X Y_{j}} & I_{X Z_{j}} \\
I_{X Z_{j}} & I_{Y Y_{j}} & I_{Y Z_{j}} \\
I_{X Z_{j}} & I_{Y Z_{j}} & I_{Z Z_{j}}
\end{array}\right]
$$

$I_{X X_{j}}, I_{X Y_{j}}, I_{X Z_{j}}, I_{Y Y_{j}}, I_{Y_{Z}}$ and $I_{Z Z_{j}}$ represent the elements of the inertial tensor the symetric matrix ${ }_{\mathrm{j}}^{\mathrm{j}} \mathrm{J}$ of each link $C_{j}$ which is expressed by the matrix (25), we have defined all these inertial parameters values in our recent work [14].

\section{The calculation of the potential energy;}

In this section, we have represented the potential energy for a manipulator arm [14], which is written by the equation (26).

$$
\mathrm{U}=\sum_{\mathrm{j}=1}^{\mathrm{n}} \mathrm{U}_{\mathrm{j}}
$$

$\mathrm{U}_{\mathrm{j}}$ : Defines the potential energy of the link $C_{j}$, which is expressed by the equation (27):

$$
\begin{aligned}
U_{j} & =-g_{0}^{T}\left(M_{j} \times{ }_{j}^{0} \mathrm{P}+{ }_{j}^{0} \mathrm{~A} \times \mathrm{MS}_{\mathrm{j}}\right) \\
& =-\left[\begin{array}{ll}
\mathrm{g}_{0}^{\mathrm{T}} & 0
\end{array}\right] \times \mathrm{T}_{0 \mathrm{j}} \times\left[\begin{array}{c}
\mathrm{MS}_{\mathrm{j}} \\
\mathrm{M}_{\mathrm{j}}
\end{array}\right]
\end{aligned}
$$

Then, we have followed the Euler-Lagrange formalism such that equation (20), we obtained the following relation (28):

$\Gamma=A(q) \ddot{q}+C(q, \dot{q}) \dot{q}+Q(q)$

where

$A(q)$ : Represents the matrix of kinetic energy $(n \times n)$, these elements are calculated as follows:

$-A_{i i}$ is equal to the coefficient of $\frac{\dot{\mathrm{q}}_{\mathrm{i}}^{2}}{2}$ located in the expression of the kinetic energy.

$-A_{i j}$ is equal to the coefficient of $\dot{q}_{i} \dot{q}_{j}$

$C(q, \dot{q}) \dot{q}$ : Defines the vector of coriolis and centrifugal forces/torques $(\mathrm{n} \times 1)$, these elements are calculated from the Christoffel symbol $c_{i, j k}$ such as system (29):

$$
\left\{\begin{array}{c}
\mathrm{c}_{\mathrm{ij}}=\sum_{\mathrm{k}=1}^{\mathrm{n}} \mathrm{c}_{\mathrm{i}, \mathrm{jk}} \dot{\mathrm{q}}_{\mathrm{k}} \\
\mathrm{c}_{\mathrm{i}, \mathrm{jk}}=\frac{1}{2}\left[\frac{\partial \mathrm{A}_{\mathrm{ij}}}{\partial \mathrm{q}_{\mathrm{k}}}+\frac{\partial \mathrm{A}_{\mathrm{ik}}}{\partial \mathrm{q}_{\mathrm{j}}}-\frac{\partial \mathrm{A}_{\mathrm{jk}}}{\partial \mathrm{q}_{\mathrm{i}}}\right]
\end{array}\right.
$$

$\mathrm{Q}(\mathrm{q})$ : Represents the vector of torques/forces of gravity, these elements are calculated as: $Q_{i}=\frac{\partial U}{\partial q_{i}}$

The elements of A, C and Q are according to the geometric and inertial parameters of the mechanism. The dynamic equations of the robot form a system of $\mathrm{n}$ differential equations of the second order, coupled and nonlinear. Then, since the inertia matrix $\mathrm{A}$ is invertible for $\mathrm{q} \in \mathcal{R}^{n}$ we may solve for the acceleration $\ddot{\mathrm{q}}$ of the manipulator as [14].

$$
\begin{gathered}
\ddot{\mathrm{q}}=\mathrm{f}(\mathrm{q}, \dot{\mathrm{q}}, \Gamma) \\
\ddot{\mathrm{q}}=-\mathrm{A}(\mathrm{q})^{-1}[\mathrm{C}(\mathrm{q}, \dot{\mathrm{q}}) \dot{\mathrm{q}}+\mathrm{Q}(\mathrm{q})-\Gamma]
\end{gathered}
$$

With,

$\mathrm{q}=\left[\begin{array}{llllll}\mathrm{q}_{1} & \mathrm{q}_{2} & \mathrm{q}_{3} & \mathrm{q}_{4} & \mathrm{q}_{5} & \mathrm{q}_{6}\end{array}\right]^{\mathrm{T}}$ : The angular position vector $(6 \times 1)$.

$\dot{q}=\left[\begin{array}{llllll}\dot{q}_{1} & \dot{q}_{2} & \dot{q}_{3} & \dot{q}_{4} & \dot{q}_{5} & \dot{q}_{6}\end{array}\right]^{T}$ : The angular velocity vector $(6 \mathrm{x} 1)$.

$\ddot{q}=\left[\begin{array}{llllll}\ddot{q}_{1} & \ddot{q}_{2} & \ddot{q}_{3} & \ddot{q}_{4} & \ddot{q}_{5} & \ddot{q}_{6}\end{array}\right]^{T}$ : The angular acceleration vector $(6 \times 1)$. $\Gamma=\left[\begin{array}{llllll}\Gamma_{1} & \Gamma_{2} & \Gamma_{3} & \Gamma_{4} & \Gamma_{5} & \Gamma_{6}\end{array}\right]^{T}:$ The input torques vector(6x1).

\subsection{Application of the input-output feedback linearization approach to a robot manipulator with six degrees of freedom:}

In this section, after we dermined the inverse dynamic model of the system [15-16], we used the equation of motion of the sixlink of the rigid manipulator robot "EPSONC4" which represented by the relation (31) and we considered the state variables of the system defined in state space as; 
W. Ghozlane et al. / Advances in Science, Technology and Engineering Systems Journal Vol. 3, No. 5, 374-381 (2018)

$$
\begin{gathered}
\mathrm{x}_{1}=\mathrm{q}_{1}, \mathrm{x}_{2}=\dot{\mathrm{q}}_{1}, \mathrm{x}_{3}=\mathrm{q}_{2}, \mathrm{x}_{4}=\dot{\mathrm{q}}_{2}, \mathrm{x}_{5}=\mathrm{q}_{3}, \mathrm{x}_{6}=\dot{\mathrm{q}}_{3}, \\
\mathrm{x}_{7}=\mathrm{q}_{4}, \mathrm{x}_{8}=\dot{\mathrm{q}}_{4}, \mathrm{x}_{9}=\mathrm{q}_{5}, \mathrm{x}_{10}=\dot{\mathrm{q}}_{5}, \mathrm{x}_{11}=\mathrm{q}_{6}, \mathrm{x}_{12}=\dot{\mathrm{q}}_{6},
\end{gathered}
$$

After derivation of the above state variables, we written the obtained system as;

$$
\left\{\begin{array}{c}
\dot{\mathrm{x}_{1}}=\mathrm{x}_{2} \\
\dot{\mathrm{x}_{2}}=\ddot{\mathrm{q}}_{1}=-\mathrm{A}\left(\mathrm{x}_{1}\right)^{-1}\left[\mathrm{C}\left(\mathrm{x}_{1}, \mathrm{x}_{2}\right) \mathrm{x}_{2}+\mathrm{Q}\left(\mathrm{x}_{1}\right)-\Gamma_{1}\right] \\
\dot{\mathrm{x}_{3}}=\mathrm{x}_{4} \\
\dot{\mathrm{x}_{4}}=\ddot{\mathrm{q}}_{2}=-\mathrm{A}\left(\mathrm{x}_{3}\right)^{-1}\left[\mathrm{C}\left(\mathrm{x}_{3}, \mathrm{x}_{4}\right) \mathrm{x}_{4}+\mathrm{Q}\left(\mathrm{x}_{3}\right)-\Gamma_{2}\right] \\
\dot{\mathrm{x}_{5}}=\mathrm{x}_{6} \\
\dot{\mathrm{x}_{6}}=\ddot{\mathrm{q}}_{3}=-\mathrm{A}\left(\mathrm{x}_{5}\right)^{-1}\left[\mathrm{C}\left(\mathrm{x}_{5}, \mathrm{x}_{6}\right) \mathrm{x}_{6}+\mathrm{Q}\left(\mathrm{x}_{5}\right)-\Gamma_{3}\right] \\
\dot{\mathrm{x}_{7}}=\mathrm{x}_{8} \\
\dot{\mathrm{x}_{8}}=\ddot{\mathrm{q}}_{4}=-\mathrm{A}\left(\mathrm{x}_{7}\right)^{-1}\left[\mathrm{C}\left(\mathrm{x}_{7}, \mathrm{x}_{8}\right) \mathrm{x}_{8}+\mathrm{Q}\left(\mathrm{x}_{7}\right)-\Gamma_{4}\right] \\
\dot{\mathrm{x}_{9}}=\mathrm{x}_{10} \\
\dot{\mathrm{x}_{10}}=\ddot{\mathrm{q}}_{5}=-\mathrm{A}\left(\mathrm{x}_{9}\right)^{-1}\left[\mathrm{C}\left(\mathrm{x}_{9}, \mathrm{x}_{10}\right) \mathrm{x}_{10}+\mathrm{Q}\left(\mathrm{x}_{9}\right)-\Gamma_{5}\right] \\
\dot{\mathrm{x}_{11}}=\mathrm{x}_{12} \\
\mathrm{x}_{12}=\ddot{\mathrm{q}}_{6}=-\mathrm{A}\left(\mathrm{x}_{11}\right)^{-1}\left[\mathrm{C}\left(\mathrm{x}_{11}, \mathrm{x}_{12}\right) \mathrm{x}_{12}+\mathrm{Q}\left(\mathrm{x}_{11}\right)-\Gamma_{6}\right]
\end{array}\right.
$$

Then, the affine form of nonlinear, multivariable and dynamic model of the robot manipulator is appeared which given by the following system (33):

$$
\left\{\begin{array}{c}
\dot{X}(t)=f(X(t))+\sum_{i=1}^{p} g_{i}(X(t)) U_{i}(t) \\
Y_{i}(t)=h_{i}(X(t)) \\
i=1,2, \ldots 6
\end{array}\right.
$$

where,

$$
\begin{aligned}
& f(x)=\left[\begin{array}{c}
x_{2} \\
-A\left(x_{1}\right)^{-1}\left[C\left(x_{1}, x_{2}\right) x_{2}+Q\left(x_{1}\right)\right] \\
x_{4} x_{3} \\
-A\left(x_{3}\right)^{-1}\left[C\left(x_{3}, x_{4}\right) x_{4}+Q\left(x_{3}\right)\right] \\
x_{6} \\
-A\left(x_{5}\right)^{-1}\left[C\left(x_{5}, x_{6}\right) x_{6}+Q\left(x_{5}\right)\right] \\
x_{8} \\
-A\left(x_{7}\right)^{-1}\left[C\left(x_{7}, x_{8}\right) x_{8}+Q\left(x_{7}\right)\right] \\
x_{10} \\
-A\left(x_{9}\right)^{-1}\left[C\left(x_{9}, x_{10}\right) x_{10}+Q\left(x_{9}\right)\right] \\
x_{12} \\
-A\left(x_{11}\right)^{-1}\left[C\left(x_{11}, x_{12}\right) x_{12}+Q\left(x_{11}\right)\right]
\end{array}\right] \\
& g_{1}(x)=\left[\begin{array}{c}
0 \\
A\left(x_{1}\right)^{-1} \\
0 \\
0 \\
0 \\
0 \\
0 \\
0 \\
0 \\
0 \\
0 \\
0
\end{array}\right] ; g_{2}(x)=\left[\begin{array}{c}
0 \\
0 \\
0 \\
A\left(x_{3}\right)^{-1} \\
0 \\
0 \\
0 \\
0 \\
0 \\
0 \\
0 \\
0
\end{array}\right] ; g_{3}(x)=\left[\begin{array}{c}
0 \\
0 \\
0 \\
0 \\
0 \\
A\left(x_{5}\right)^{-1} \\
0 \\
0 \\
0 \\
0 \\
0 \\
0
\end{array}\right]
\end{aligned}
$$

$$
g_{4}(x)=\left[\begin{array}{c}
0 \\
0 \\
0 \\
0 \\
0 \\
0 \\
0 \\
A\left(x_{7}\right)^{-1} \\
0 \\
0 \\
0 \\
0
\end{array}\right] ; g_{5}(x)=\left[\begin{array}{c}
0 \\
0 \\
0 \\
0 \\
0 \\
0 \\
0 \\
0 \\
0 \\
A\left(x_{9}\right)^{-1} \\
0 \\
0
\end{array}\right] ; g_{6}(x)=\left[\begin{array}{c}
0 \\
0 \\
0 \\
0 \\
0 \\
0 \\
0 \\
0 \\
0 \\
0 \\
0 \\
A\left(x_{11}\right)^{-1}
\end{array}\right]
$$

And,

$$
\begin{aligned}
& X=\left[x_{1}, x_{2}, x_{3}, x_{4}, x_{5}, x_{6} x_{7}, x_{8}, x_{9}, x_{10}, x_{11}, x_{12}\right]^{T} ; \\
& \dot{X}=\left[\dot{x}_{1}, \dot{x}_{2}, \dot{x}_{3}, \dot{x}_{4}, \dot{x}_{5}, \dot{x}_{6} \dot{x}_{7}, \dot{x}_{8}, \dot{x}_{9}, \dot{x}_{10}, \dot{x}_{11}, \dot{x}_{12}\right]^{T} ; \\
& U=\left[u_{1}, u_{2}, u_{3}, u_{4}, u_{5}, u_{6}\right]^{T}=\left[\Gamma_{1}, \Gamma_{2}, \Gamma_{3}, \Gamma_{4}, \Gamma_{5}, \Gamma_{6}\right]^{T} ;
\end{aligned}
$$

$$
\text { Links positions }\left\{\begin{array}{l}
y_{1}=h_{1}(x)=x_{1}=q_{1} \\
y_{2}=h_{2}(x)=x_{3}=q_{2} \\
y_{3}=h_{3}(x)=x_{5}=q_{3} \\
y_{4}=h_{4}(x)=x_{7}=q_{4} \\
y_{5}=h_{5}(x)=x_{9}=q_{5} \\
y_{6}=h_{6}(x)=x_{11}=q_{6}
\end{array}\right.
$$

So, we made the derivation of each output $y_{i}$ intel the inputs appeared in the expression and we computed the relative degrees $r_{i}$ for each joint of the robot as follows [17-18];

$$
\left\{\begin{array}{c}
y_{1}=h_{1}(x)=x_{1} \\
\dot{y}_{1}=L_{f} h_{1}(x)=\dot{x}_{1}=x_{2} \\
y_{1}{ }^{(2)}=L_{f}{ }^{2} h_{1}(x)+L_{g} L_{f} h_{1}(x) u \\
r_{1}=2 \\
y_{2}=h_{2}(x)=x_{3} \\
\dot{y}_{2}=L_{f} h_{2}(x)=\dot{x}_{3}=x_{4} \\
y_{2}{ }^{(2)}=L_{f}{ }^{2} h_{2}(x)+L_{g} L_{f} h_{2}(x) u \\
r_{2}=2 \\
\vdots \\
y_{6}=h_{6}(x)=x_{11} \\
\dot{y}_{6}=L_{f} h_{6}(x) \dot{x}_{11}=x_{12} \\
y_{6}{ }^{(2)}=L_{f}{ }^{2} h_{6}(x)+L_{g} L_{f} h_{6}(x) u \\
r_{6}=2
\end{array}\right.
$$

Therefore, the relative degree of each joint $r_{i}$ is well defined and is equal to 2 , so we computed the nonlinear control law $u_{i}(t)$ of each joint of the system as this relation (36);

$$
\begin{gathered}
u_{i}(x(t))=-\frac{L_{f}^{r_{i}} h_{i}(x(t))}{L_{g}^{r^{-1}} L_{f} h_{i}(x(t))}+\frac{v_{i}(t)}{L_{g}^{r^{-1}} L_{f} h_{i}(x(t))} \\
i=1 \ldots 6
\end{gathered}
$$

By using the nonlinear control law and diffeomorphic transformation given above, the nonlinear dynamic system with six degrees of freedom is converted into the following Brunovesky canonical form and simultaneously output decoupled [19]. 


$$
\left\{\begin{array}{c}
\dot{Z}=A z+B V \\
Y=C Z
\end{array}\right.
$$

where,

$$
\begin{aligned}
& Z=\left[\begin{array}{c}
z_{1} \\
z_{2} \\
\vdots \\
z_{12}
\end{array}\right]=\left[\begin{array}{c}
h_{1} \\
L_{f} h_{1} \\
h_{2} \\
L_{f} h_{2} \\
\cdot \\
\vdots \\
h_{6} \\
L_{f} h_{6}
\end{array}\right]=\left[\begin{array}{c}
x_{1} \\
x_{2} \\
\vdots \\
x_{12}
\end{array}\right] ; \dot{Z}=\left[\begin{array}{c}
\dot{z}_{1} \\
\dot{z}_{2} \\
\vdots \\
\dot{z}_{12}
\end{array}\right]=\left[\begin{array}{c}
\dot{x}_{1} \\
\dot{x}_{2} \\
\vdots \\
\dot{x}_{12}
\end{array}\right] ; \mathrm{V}=\left[\begin{array}{c}
\mathrm{v}_{1} \\
\mathrm{v}_{2} \\
\vdots \\
\mathrm{v}_{6}
\end{array}\right] \\
& \mathrm{A}=\left[\begin{array}{cccccccc}
0 & 1 & & & & & & \\
0 & 0 & & \cdots & & & 0 & \\
\ddots & & \ddots & \ldots & & & \\
& & \ldots & 1 & & & \vdots \\
& & \cdots & 0 & 0 & & & \\
& & & \ddots & & \ddots & & \vdots \\
\vdots & & & & \ddots & \ddots & \vdots \\
\vdots & & \cdots & & & \ddots & \vdots \\
0 & & & & \cdots & 0 & 1 \\
& & & & & 0 & 0
\end{array}\right]
\end{aligned}
$$

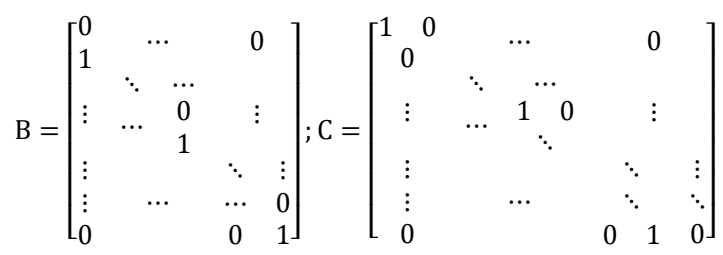

The above matrices $\mathrm{A}, \mathrm{B}$ and $\mathrm{C}$ are of dimension respectively : $(12 \times 12),(12 \times 6)$ and $(6 \times 12)$.

We note that, the obtained linear system (37) definied by six decoupled and linear subsystems that is has the following form (38), and $\mathrm{i}=1 \ldots 6$;

$$
\left\{\begin{array}{c}
\dot{z}_{i}=\left[\begin{array}{ll}
0 & 1 \\
0 & 0
\end{array}\right] z_{i}+\left[\begin{array}{l}
0 \\
1
\end{array}\right] v_{i} \\
y_{i}=\left[\begin{array}{ll}
1 & 0
\end{array}\right] z_{i}
\end{array}\right.
$$

where,

$$
z_{i}=\left[\begin{array}{c}
Z_{2 i-1} \\
z_{2 i}
\end{array}\right]
$$

For the objective of linear control, we added to each subsystem a linear PD controller which has represented equation (39) as;

$$
\begin{gathered}
v_{i}(t)=K_{p}^{i}\left(1+K_{d}^{i} s\right) e_{i}(t) ; i=1 \ldots 6 \\
e_{i}(t)=y_{d_{i}}(t)-y_{i}(t) \\
v_{i}=y_{i}^{r_{i}}
\end{gathered}
$$

we obtained the relative degree for each subsystem as : $r_{i}=2$

$$
v_{i}=K_{d}^{i}\left(y_{d_{i}}{ }^{\left(r_{i}-1\right)}-y_{i}{ }^{\left(r_{i}-1\right)}\right)+K_{p}^{i}\left(y_{d_{i}}-y_{i}\right)
$$

$$
v_{i}(t)=\left[\begin{array}{ll}
K_{p}^{i} & K_{d}^{i}
\end{array}\right]\left[\begin{array}{c}
y_{d_{i}}(t)-y_{i}(t) \\
y_{d_{i}}{ }^{\left(r_{i}-1\right)}(t)-y_{i}{ }^{\left(r_{i}-1\right)}(t)
\end{array}\right]
$$

Then, we used the diffeomorphic transformation (16) presented above, we considered these relations for each SISO subsystem:

$$
\left\{\begin{array}{c}
z_{1}^{i}=h_{i}(x(t))-y_{d_{i}} \\
z_{2}^{i}=L_{f} h_{i}(x(t))-\dot{y}_{d_{i}}
\end{array}\right.
$$

That is leads to the non-linear coordinate transformation given by:

$$
\begin{gathered}
\left\{\begin{array}{c}
\dot{z}_{1}^{i}=z_{2}^{i}=L_{f} h_{i}(x(t))-\dot{y}_{d_{i}} \\
\dot{z}_{2}^{i}=L_{f}{ }^{2} h_{i}(x(t))+L_{g} L_{f} h_{i}(x(t)) u_{i}(x(t))-\ddot{y}_{d_{i}}
\end{array}\right. \\
v_{i}(t)=-\sum_{j=0}^{r_{i}-1} K_{j} Z_{i}=-\sum_{j=0}^{r_{i}-1} K_{j}\left[L_{f}{ }^{(j)} h_{i}(x(t))-y_{d_{i}}{ }^{(j)}(t)\right]
\end{gathered}
$$

So, we expressed the relation (46) in equation (36),we obtained:

$$
u_{i}(x(t))=-\frac{L_{f}{ }^{r_{i}} h_{i}(x(t))}{L_{g}{ }^{r_{i}-1} L_{f} h_{i}(x(t))}+\frac{-\sum_{j=0}^{r_{i}-1} K_{j}\left[L_{f}{ }^{(j)} h_{i}(x(t))-y_{d_{i}}{ }^{(j)}(t)\right]}{L_{g}{ }^{r_{i}-1} L_{f} h_{i}(x(t))} \text { (47) }
$$

Next, we expressed the equation (47) in the subsystem (45), the non linear subsystem (45) was transformed to a linear subsystem which expressed as relation (48):

$$
\begin{gathered}
{\left[\begin{array}{c}
\dot{z}_{1}^{i} \\
\dot{z}_{2}^{i}
\end{array}\right]=\left[\begin{array}{cc}
0 & 1 \\
K_{p}^{i} & K_{d}^{i}
\end{array}\right]\left[\begin{array}{l}
z_{1}^{i} \\
z_{2}^{i}
\end{array}\right]} \\
i=1 \\
i=6
\end{gathered}
$$

The obtained linear subsystem consists of a second order system with linear output, that is can be computed the poles of the above subsystem as;

$$
s_{1,2}=-\xi \omega_{n} \mp \omega_{n} \sqrt{1-\xi^{2}}
$$

Where, $\xi$ : means damping ratio, $\omega_{n}$ :means natural frequency, for goal of stability we chosed the parameters of each PD controller applied to each subsystem as [19]:

$$
\left\{\begin{array}{l}
K_{p}^{j}=\omega_{n}{ }^{2} \\
K_{d}^{j}=\xi \omega_{n}
\end{array} j=1 \ldots 6\right.
$$

\section{Simulation Results}

In order to improve the efficiency of the proposed approach, we applied the above method of linearization to a robot manipulator which represent a non linear, decoupled and multivariable system. We imposed the sinusoidal signals as $y_{i d}(t), \dot{y}_{i d}(t)$ and $y_{i d}{ }^{r_{i}}(t)$ which are represented inputs desired trajectories to each joint of the studied system. Therefore, the relative degrees $r_{i}$ is well 
defined, we presented the simulation results depicting the output $y_{i}{ }^{r_{i}}(t)$ of each joint $\mathrm{i}=1 \ldots 6$ as shown in Figures 2,3,4,5,6,7,8-9.
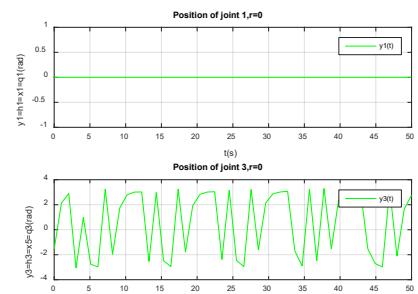

Position of tisint 5, ren
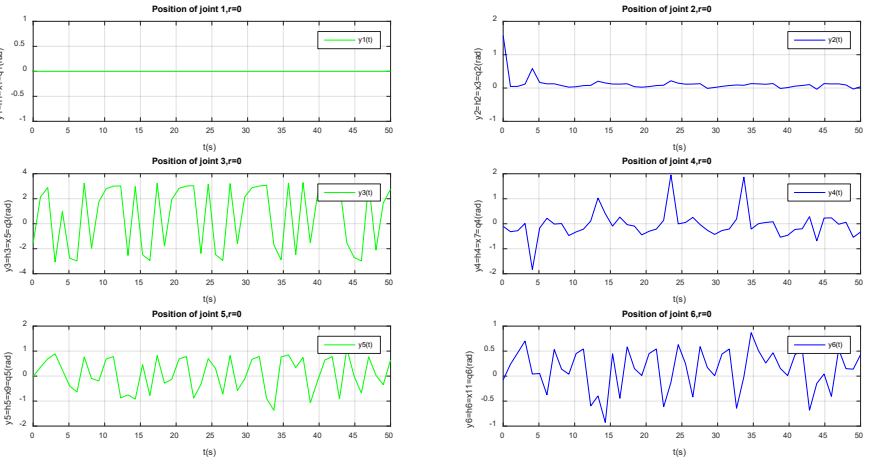

Position of tijint t,,$=0$

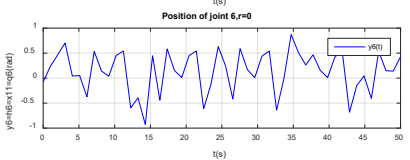

Figure 2. The outputs $y_{i}{ }^{r_{i}}(t)$ plot of each joint, the relative degrees $r_{i}=0$, $\mathrm{i}=1 . . .6$.

Firstly, we made the simulation of the outputs of each joint of the robot, $y_{i}(t)=h_{i}(x(t))=x_{i}(t)$, the results are therefore given in Figure 2, we can see the non linearity of each subsystem. Second, we applied the Lie derivation to each output we shown the results presented in Figure 3 as.
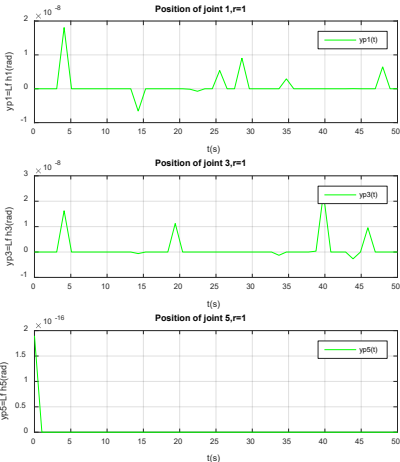
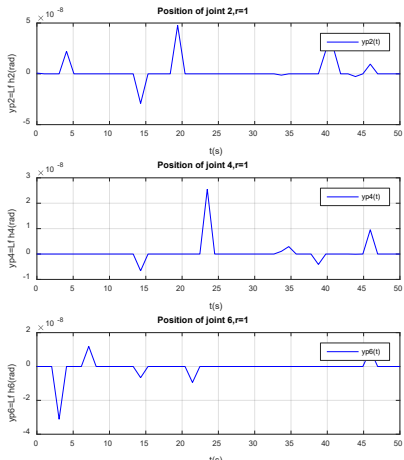

Figure 3. The outputs $y_{i}{ }^{r}(t)$ plot of each joint, the relative degrees $r_{i}=1$, $i=1 \ldots 6$.

In this case, the relative degrees equal to $r_{i}=$ $1, L_{g} L_{f} h_{i}(x(t))=0$, so no one input has been appeared, as shown in Figure 3, the system still non linear. Finally, we made the derivation again of each recent output $\dot{y}_{l}$ the inputs appeared in the expression and we computed the final relative degrees $r_{i}=$ 2 for each joint of the robot, $y_{i}^{(2)}=L_{f}{ }^{2} h_{i}(x)+$ $L_{g} L_{f} h_{i}(x) u, L_{g} L_{f} h_{i}(x) \neq 0$, the results was illustrated in these Figures 4,5,6,7,8-9.

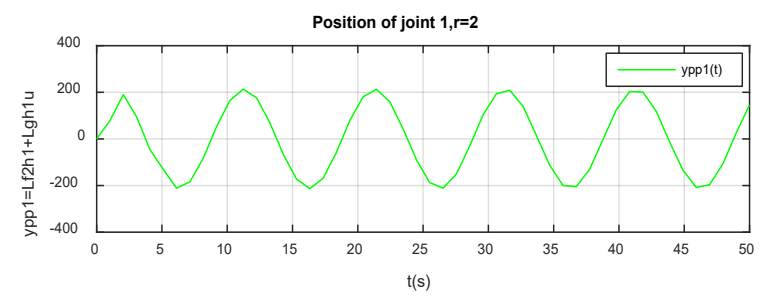

Figure 4. The output $y_{i}{ }^{r_{i}}(t)$ plot of the joint 1 , the relative degree $r_{1}=2$.

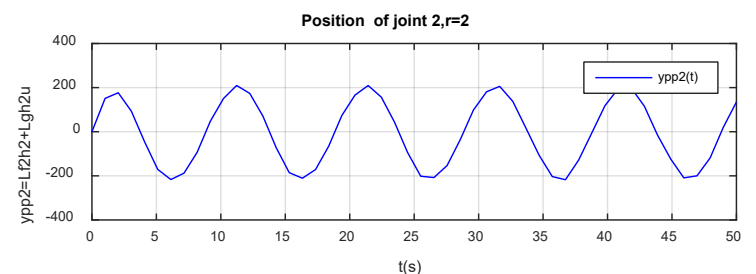

Figure 5. The output $y_{i}{ }^{r_{i}}(t)$ plot of the joint 2 , the relative degree $r_{2}=2$.

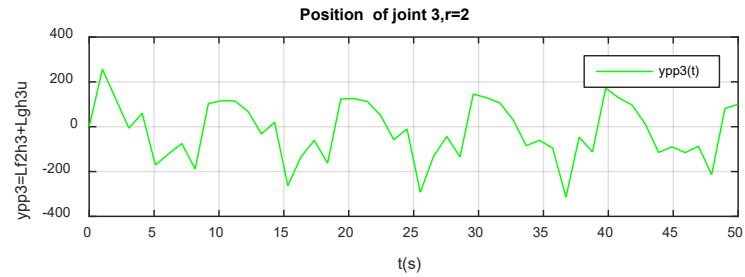

Figure 6 . The output $y_{i}{ }^{r_{i}}(t)$ plot of the joint 3 , the relative degree $r_{3}=2$.

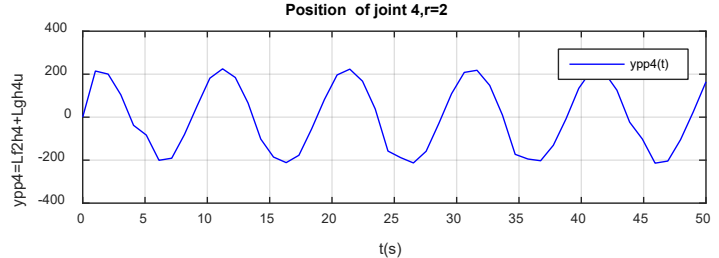

Figure 7. The output $y_{i}{ }^{r_{i}}(t)$ plot of the joint 4 , the relative degree $r_{4}=2$.

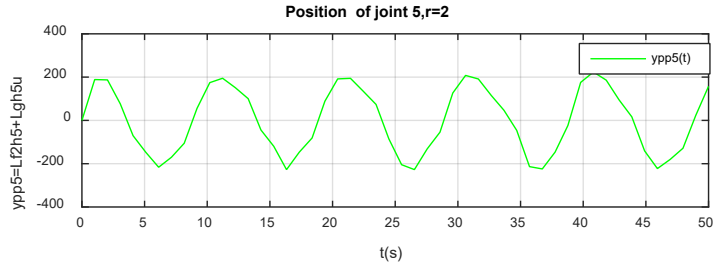

Figure 8 . The output $y_{i}{ }^{r_{i}}(t)$ plot of the joint 5 , the relative degree $r_{5}=2$.

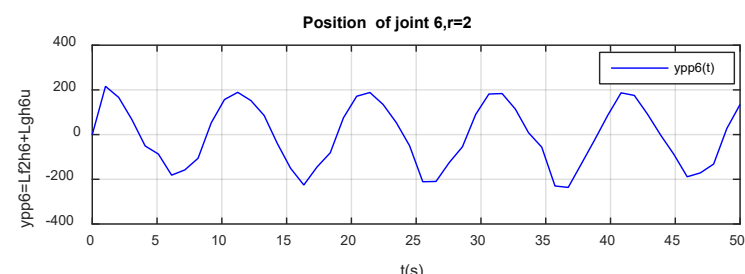

Figure 9. The output $y_{i}{ }^{r_{i}}(t)$ plot of the joint 6 , the relative degree $r_{6}=2$.

These Figures 4,5,6,7,8-9 shown that the output of each SISO subsystem asymptotically tracks the sinusoidal input trajectory with minimum of oscillation and the efficiency of the studied approach.

\section{Conclusion}

In this paper, the input-output feedback linearization approach is presented which is a way of algebraically transforming nonlinear, multivaribles, complex and dynamics systems into linear ones, so we can be applied linear control, this technique has attracted lots of research in recent years. For that we applied this proposed approach to derive the control of a robot manipulator with six degrees of freedom which followed two major steps. Firstly, by using the above approach and 
diffeomorphic transformation, we converted the non linear and decoupled dynamics model of the robot to linear system. Then, we designed a linear PD control law for each decoupled subsystem to control the angular position of each joint of this robot for tracking purposes. Finally, the obtained results in different simulations illustrated the accuracy of the proposed approach.

\section{References}

[1] MW. Spong, S.Hutchinson, and M. Vidyasagar. Robot Dynamics and Control, Second Edition. January 28, 2004.

[2] J.-J. Slotine \& W. Li, Applied Nonlinear Control, Prentice-Hall, Englewood Cliffs, NJ, 1991.

[3] A. Isidori. Nonlinear Control Systems. Springer Verlag, 1989.

[4] H. Khalil. Nonlinear Systems. Prentice Hall, Upper Saddle River, Second Edition, 1996.

[5] J.Hauser,S.Sastry, and P.Kokolovic,"Non linear control via approximate Input_Output Linearization, The Ball and Beam Exemple". IEEE Transactions onAutomatic Control,Vol 37,No.3 March 1992.

[6] E.D. Sontag,M. Thoma,A. Isidori and J.H. van Schuppen. Nonlinear and Adaptive Control with Applications, 2008 Springer-Verlag London Limited.

[7] H. Nijmeijer and A. J. van der Schaft, Nonlinear Dynamical Control Systems, Springer-Verlag, New York, 1990.

[8] M. Vidyasagar. Nonlinear Systems Analysis. Prentice Hall,Second Edition, 1993.

[9] K.M. Hangos, J. Bokor et G. Szederkényi, Analysis and Control of Nonlinear Process Systems, Springer-Verlag London Limited, 2004.

[10] J.HAUSER, SH. SASTRY and G. MEYER. "Nonlinear Control Design for Slightly Non minimum Phase Systems: Application to V/STOL Aircraft". International Federation of Automatic Control. Vol. 28, No. 4, pp. 665-679, 1992.

[11] W.Ghozlane and J.Knani."Modelling and Simulation Using Mathematical and CAD Model of a Robot with Six Degrees of Freedom,"CEIT-2016,

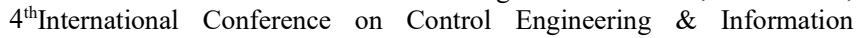
Technology on IEEE.16-18 December 2016-Hammamet, Tunisia.

[12] B.Siciliano and O. Khatib, Handbook of Robotics: Springer, 2007.

[13] W. Khalil and E. Dombre, Modeling Identification and Control of Robots, Hermes Penton London, 2002.

[14] Thomas R. Kurfess, Robotics and Automation Handbook, CRC press, 2005.

[15] Y . L . Chen: " Nonlinear Feedback and Computer Control of Robot Arms". D.Sc. Dissertation, Washington University, St. Louis 1984.

[16] V.D. Yurkevich, Design of Nonlinear Control Systems with the Highest Derivative in Feedback,World Scientific Publishing, 2004.

[17] Frank L.Lewis, Robot dynamics and control, in robot Handbook: CRC press, 1999.

[18] L. Sciavicco and B. Siciliano, Modeling and control of robot manipulators, 2nd edition, Springer-Verlag London Limited, 2000.

[19] C.T.Leondes. Control and Dynamic Systems,Advances In Theory and Applications, part 1 of 2,USA,1991.

[20] Zakaria, M. Z., Jamaluddin, H., Ahmad, R. \& Loghmanian, S. M. R. (2010). "Multiobjective Evolutionary Algorithm Approach in Modeling DiscreteTime Multivariable Dynamics Systems". Computational Intelligence, Modelling and Simulation (CIMSiM), 2010 Second International Conference on, Bali, Indonesia. 28-30 Sept. 2010. 65-70.

[21] Zakaria, M. Z., Jamaluddin, H., Ahmad, R. \& Loghmanian, S. M. R. (2012). "Comparison between Multi-Objective and Single-Objective Optimization for the Modeling of Dynamic Systems". Journal of Systems and Control Engineering, Part I. Proc. Instn. Mech. Engrs., 226 (7): 994-1005. 